\title{
DISEÑO DE VIGAS DE GRAN PERALTE CON ALTO NIVEL DE ASIMETRÍA USANDO LA TÉCNICA DEL PUNTAL TIRANTE
}

\section{DESIGN OF LARGE BEAM BEAMS WITH HIGH LEVEL OF ASYMMETRY USING THE STRUT BRACE TECHNIQUE}

\author{
Glenda Milagros, Linaja García ${ }^{1}$ \\ Dina Marlene Cotrado Flores ${ }^{2}$
}

Aceptado: 09/05/2020

Publicado online: 14/12/2020

\begin{abstract}
RESUMEN
Se estudia las vigas de gran peralte, que son usadas para soportar grandes cargas o por condiciones arquitectónicas. Esta investigación consiste en la determinación del diseño de una viga discontinua en totalidad, tanto en carga como en geometría, usando la técnica puntal - tirante, por lo cual, usando información bibliográfica de trabajos de investigación similares se procedió a la realización del diseño estructural de esta viga, la técnica es recomendada por la norma peruana E.060. Posteriormente, se realizó el modelado en el software AbaqusCAE para poder determinar esfuerzos del refuerzo de acero, porcentaje de parámetros de daño, esfuerzos de Von Mises y Tresca, porcentaje de fisuras y porcentaje de aplastamiento, obteniendo como resultado un buen diseño para este tipo de estructuras, que fue verificado en el software donde tiene un buen comportamiento, concluyendo que, para la muestras con discontinuidades considerables se recomienda usar la técnica del Strut and Tie. 3
\end{abstract}

Palabras clave: desplazamiento, distribución de esfuerzos de von mises, distribución de esfuerzos de tresca, fisuras, parámetros de daño, porcentaje de aplastamiento

\begin{abstract}
It studies the beams of great can't, which are used to withstand large loads or for architectural conditions. This investigation consists in the determination of the design of a discontinuous beam in totality, both in load and in geometry, using the strut-pull technique, therefore, using bibliographic information of similar research works, the structural design of this beam, the technique is recommended by the Peruvian Standard E.060. Subsequently, modeling was carried out in the AbaquSCAE software to determine steel reinforcement efforts, percentage of damage parameters, Von Mises and Tresca efforts, percentage of cracks and crushing percentage, resulting in a good design for this type of structures, which was verified in the software where it has a good behavior, concluding that, for samples with considerable discontinuities it is recommended to use the Strut and Tie technique.
\end{abstract}

Keywords: displacement, von mises stress distribution, tresca stress distribution, fissures, damage parameters, crush percentage

\footnotetext{
${ }^{1}$ Maestro en Ingeniería Civil con mención en Estructuras de la Universidad Privada de Tacna, EPS Tacna, Ingeniero Asistente en GMI, Provías Descentralizado, glendalg15@gmail.com. (D) 0000-0002-1100-1733

2 Ingeniera Civil y maestra con mención en estructuras de la Universidad Privada De Tacna, con estudios culminados de segunda especialidad en Ingeniería sismoresistente en la Universidad Nacional de Ingeniería (UNI).dcotrado@upt.edu.pe 


\section{INTRODUCCIÓN}

Esta investigación consiste en la determinación del diseño de una viga discontinua en totalidad, usando la técnica puntal - tirante también conocida Strut and Tie, por lo cual, se realizó el diseño estructural de una viga con asimetría tanto geométrica como en carga, la técnica ya mencionada es recomendada por la norma peruana E.060. Se realizó el modelado en el software AbaqusCAE para poder determinar esfuerzos del refuerzo de acero, porcentaje de parámetros de daño, esfuerzos de Von Mises y Tresca, porcentaje de fisuras y porcentaje de aplastamiento, obteniendo como resultado un buen diseño para este caso de estructuras. En Tacna, no hay casos estudiados de análisis y diseño de vigas de gran peralte, ya que en nuestra ciudad la mayoría de edificaciones son viviendas unifamiliares y multifamiliares, en su mayoría de carácter informal. Perú es un país que es considerado una zona sísmica y esto demanda diseños que garanticen la seguridad de las estructuras, en este caso aplicados al elemento concreto armado, por esta razón las vigas altas o también conocidas como vigas de gran peralte son recomendables usarlas en estructuras que soporten grandes cargas y grandes luces. La norma E.060 no brinda mucha información con respecto a un buen diseño de estas, pues contribuye a juntar criterios de diseño (Contreras, 2010).

Chowdhury (2014), estudió el comportamiento de una viga con dos agujeros. Se utilizó un método de elementos finitos numéricos no lineales tridimensionales utilizando ANSYS 10,0. García (2009) encontró una base en el modelo de Hwang et al. (2001). A pesar de que el modelo propuesto se basa en los conceptos de estos autores, éste es completamente diferente en la manera en que determina el esfuerzo último de corte de los muros cortos de concreto armado. Young (2009), desarrolló nomogramas y demostró su aplicabilidad para lo simple diseño de haz profundo admitido para simplificar una serie de pasos de cálculo. Para este propósito, se aplicaron modificaciones básicas de la formulación del modelo Strut-Tie para la viga de diseño de gran luz, brevemente descrito. García G. L. (2019), describió el comportamiento de una viga de gran peralte empleando técnicas modernas frente a las exigencias de la norma peruana de concreto armado, a través del diseño por dos técnicas, la técnica de Strut and Tie y la técnica de la norma peruana E.060, la muestra estudiada, presenta el estudio de una viga simétrica. Loayza (2015), propuso expresiones para obtener la resistencia probable a flexo compresión de muros de concreto armado con base en un trabajo estadísticamente calibrado con resultados experimentales, los cuales fueron seleccionados luego de revisar de manera crítica la literatura existente.

Se denomina a vigas de gran peralte a aquellas estructuras que la relación de luz/profundidad entre vigas simplemente apoyadas sean menor a 2, o en vigas continuas 2.5 (RNE, 2009). El inicio de la técnica puntal-tirante es un modelo enrejado de barras para representar vigas agrietadas con el fin de explicar el mecanismo de resistencia al corte de vigas con armadura longitudinal y estribos, en el cual se considera la interacción entre puntales de hormigón en compresión y el refuerzo longitudinal y transversal a tracción. (S.R.Chowdhury, 2014). El concreto es una combinación de material pétreo, cemento y agua, sin embargo, agregándole acero de refuerzo embebidas al concreto permite mejorar la resistencia (Arkiplus, 2019).

Su aplicabilidad no sólo comprende estructuras planas, sino que se extiende a otro tipo de estructuras.

\section{METODOLOGÍA}

La variable Independiente: Vigas de gran Peralte con alto nivel de asimetría de concreto armado. Dimensiones de estudio: Geometría del elemento estructural y Resistencia de compresión del concreto y esfuerzo de fluencia del acero de refuerzo. La variable dependiente: Técnica puntal tirante. Dimensiones de estudio: Propuesta de armadura, distribución de esfuerzos y porcentaje de parámetros de daño. El tipo de estudio que se evaluó fue explicativo. El nivel de investigación 
al que se llegó según Hurtado (2006) es comprensivo. Debido a que el análisis y diseño de vigas de gran peralte, es un campo poco estudiado en la región de Tacna, cuyos objetivos son analizar y comparar corresponde a un nivel de investigación aprehensivo. Población de estudio: Las vigas asimétricas de gran peralte. La muestra: La viga asimétrica de gran peralte a estudiar se muestra en la siguiente imagen, las medidas mostradas son en metros.

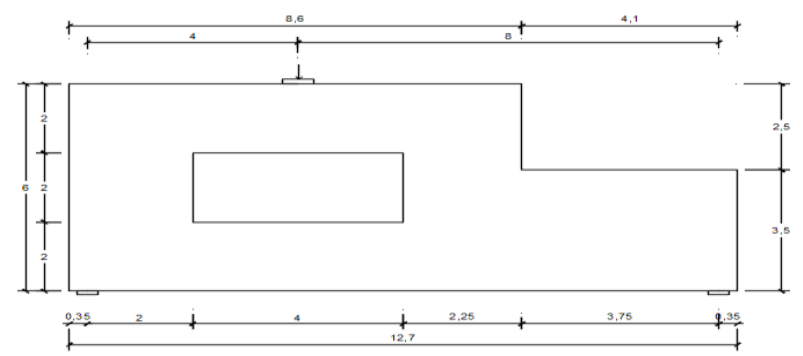

Figura 1 Muestra estudiada en la presente investigación.

\section{PROCEDIMIENTO, TÉCNICAS E INSTRUMENTOS}

Diseño según la Técnica Puntal Tirante se muestra el procedimiento de la técnica puntal tirante de la muestra estudiada.

Tabla 1

Propiedades de los materiales.

MATERIALES

\begin{tabular}{lll}
\hline Concreto & 300 & $\mathrm{~kg} / \mathrm{cm} 2$ \\
\hline Acero & 4200 & $\mathrm{~kg} / \mathrm{cm} 2$ \\
\hline
\end{tabular}

Tabla 2

Dimensiones de las placas de apoyo.

\begin{tabular}{lll}
\hline \multicolumn{3}{c}{ DIMENSIONES } \\
\hline Placas & Base & Ancho \\
\hline De apoyo & 0.4 & 0.35 \\
\hline De carga & 0.6 & 0.35 \\
\hline Viga & - & 0.35 \\
\hline
\end{tabular}

a. Identificación de Regiones: Toda la estructura corresponde a una región discontinua, ya que presenta asimetría en geometría y en cargas que es notable a simple vista.

b. Cálculo de las fuerzas Actuantes en las zonas discontinuas: Las fuerzas actuantes en este caso son las reacciones de los apoyos, la reacción 1 y 2 . Se calcularon por condiciones de equilibrio.

c. Elección de la armadura: En la figura, la que finalmente es la escogida para el desarrollo de este ejemplo, soluciona el problema de la viga inferior con la incorporación de tensores en la porción superior de esta. (Adm, 2017).

d. Geometría general: Una vez elegido la propuesta de armadura, se procede a hallar cada una de las fuerzas actuantes en esta, considerando las mismas condiciones que se tiene para una armadura normal. Para ello se optó por ponerles un nombre a cada barra de esta armadura.

e. Esfuerzo en puntales y tirantes: Los resultados se mostrarán en los siguientes cuadros.

Tabla 3

Ángulos según la técnica propuesta de Puntal-tirante.

\begin{tabular}{ccc}
\hline \multicolumn{3}{l}{ ANGULOS } \\
\hline \$1 & 35.923 & $\circ$ \\
\hline$\phi 2$ & 57.529 & $\circ$ \\
\hline \$3 & 32.898 & $\circ$ \\
\hline \$4 & 40.778 & $\circ$ \\
\hline
\end{tabular}

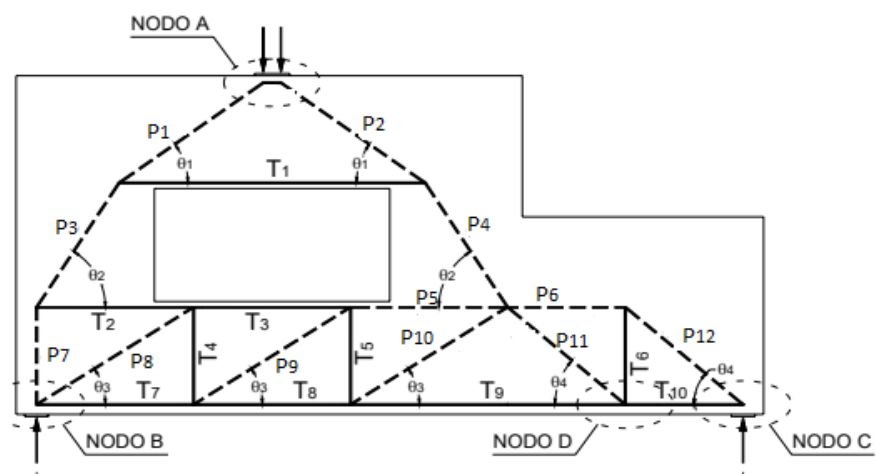

Figura 2 Geometría general de la propuesta de armadura. 


\section{Tabla 4}

Fuerzas calculados.

\begin{tabular}{|c|c|c|}
\hline \multicolumn{3}{|c|}{ FUERZAS } \\
\hline$F u$ & 180 & $\mathrm{Tn}$ \\
\hline$F 1$ & 90 & $\mathrm{Tn}$ \\
\hline$F 2$ & 90 & $\mathrm{Tn}$ \\
\hline$R 1$ & 120 & Tn \\
\hline$R 2$ & 60 & $\mathrm{Tn}$ \\
\hline \multicolumn{3}{|c|}{ PUNTALES } \\
\hline PO & 124.225 & $\mathrm{Tn}$ \\
\hline P1 & 153.401 & $\mathrm{Tn}$ \\
\hline P2 & 153.401 & $\mathrm{Tn}$ \\
\hline P3 & 106.678 & Tn \\
\hline P4 & 106.678 & $\mathrm{Tn}$ \\
\hline P5 & 35.481 & $\mathrm{Tn}$ \\
\hline P6 & 69.565 & $\mathrm{Tn}$ \\
\hline P7 & 900 & $\mathrm{Tn}$ \\
\hline P8 & 55.234 & $\mathrm{Tn}$ \\
\hline P9 & 55.234 & $\mathrm{Tn}$ \\
\hline P10 & 55.234 & $\mathrm{Tn}$ \\
\hline P11 & 91.866 & $\mathrm{Tn}$ \\
\hline P12 & 91.866 & $\mathrm{Tn}$ \\
\hline P7-8 & 128.65 & $\mathrm{Tn}$ \\
\hline \multicolumn{3}{|c|}{ TIRANTES } \\
\hline T1 & 66.953 & Tn \\
\hline T2 & 57.273 & $\mathrm{Tn}$ \\
\hline T3 & 10.896 & $\mathrm{Tn}$ \\
\hline T4 & 30 & Tn \\
\hline T5 & 30 & $\mathrm{Tn}$ \\
\hline T6 & 60 & $\mathrm{Tn}$ \\
\hline T7 & 46.377 & $\mathrm{Tn}$ \\
\hline $\mathrm{T} 8$ & 92.754 & $\mathrm{Tn}$ \\
\hline T9 & 139.13 & Tn \\
\hline T10 & 69.565 & $\mathrm{Tn}$ \\
\hline
\end{tabular}

f. Dimensionamiento de Puntales, Tirantes y zonas nodales:

\section{Verificación de apoyos}

\section{Placa de Carga Nodo A}

El área de la placa es $A_{c}=60 \mathrm{~cm} * 35 \mathrm{~cm}=2100 \mathrm{~cm}^{2}$

El esfuerzo portante en el punto de carga es: $=85.7 \mathrm{~kg} / \mathrm{cm}^{2}$

Las placas de los apoyos tienen un comportamiento de CCC (compresión- compresióncompresión), entonces la resistencia efectiva a compresión es: $=255 \mathrm{~kg} / \mathrm{cm}^{2}$

Una vez calculado el esfuerzo portante y la resistencia efectiva, tienen que cumplir la siguiente condición: $191.25 \mathrm{~kg} / \mathrm{cm}^{2}>85.7 \mathrm{~kg} / \mathrm{cm}^{2}$ ¡OK! La condición cumple.

Con esta condición se puede afirmar que la placa en el nodo A, soporta la carga solicitada.

Placa de Apoyo Nodos $B$ y $C$

El área de la placa es $A_{c}=40 \mathrm{~cm} * 35 \mathrm{~cm}=1400 \mathrm{~cm}^{2}$

El esfuerzo portante en el punto de carga es: $85.7 \mathrm{~kg} / \mathrm{cm}^{2}$

Las placas de los apoyos tienen un comportamiento de CCT (compresión- compresióntracción), entonces la resistencia efectiva a compresión es: $204 \mathrm{~kg} / \mathrm{cm}^{2}$

Una vez calculado el esfuerzo portante y la resistencia efectiva, tienen que cumplir la siguiente condición: $153 \mathrm{~kg} / \mathrm{cm}^{2}>85.7 \mathrm{~kg} / \mathrm{cm}^{2}$ iOK! La condición cumple.

Con esta condición se puede afirmar que la placa en el nodo B y C, soporta la carga solicitada. 


\section{Verificación de zona nodales}

La zona nodal A1.

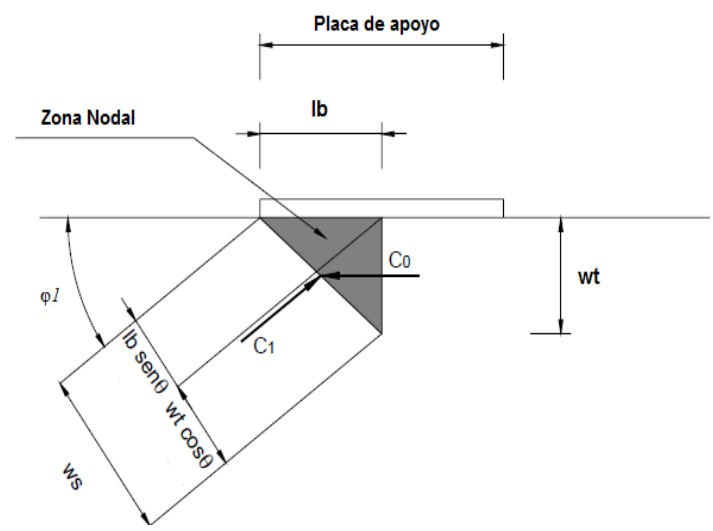

Figura 3 Zona nodal A1.

Para poder calcular los anchos de cada fuerza aplicada en ese nodo. Donde lb, es la mitad del ancho de la placa, $0.30 \mathrm{~m}$, wt es el ancho efectivo del tirante, y ws es el ancho efectivo del puntal. Recordar que esta zona nodal tiene un comportamiento CCC. $w_{s}=0.378 m$

Entonces con esto se concluye que el ancho de la fuerza $\mathrm{C} 1$, es $0.378 \mathrm{~m}$, el ancho de Co es $0.25 \mathrm{~m}$ y el ancho de la carga es $0.30 \mathrm{~m}$.

De la misma forma se hace para la zona nodal B. Para poder calcular los anchos de cada fuerza aplicada en ese nodo. Donde lb, es la mitad del ancho de la placa, $0.40 \mathrm{~m}$, wt es el ancho efectivo del tirante, $0.35 \mathrm{~m}$, y ws es el ancho efectivo del puntal. Recordar que esta zona nodal tiene un comportamiento CCT. $w_{s}=0.499 \mathrm{~m}$

Entonces con esto se concluye que el ancho de la fuerza C7, es $0.499 \mathrm{~m}$, el ancho de T7 es $0.35 \mathrm{~m}$ y el ancho de la carga es $0.40 \mathrm{~m}$.

Por último, la zona nodal C. Para poder calcular los anchos de cada fuerza aplicada en ese nodo, que son los ángulos calculados de toda la propuesta de armadura. Donde lb, es la mitad del ancho de la placa, $0.40 \mathrm{~m}$, wt es el ancho efectivo del tirante, $0.35 \mathrm{~m}$, y ws es el ancho efectivo del puntal. Recordar que esta zona nodal tiene un comportamiento CCT. $w_{s}=0.526 \mathrm{~m}$

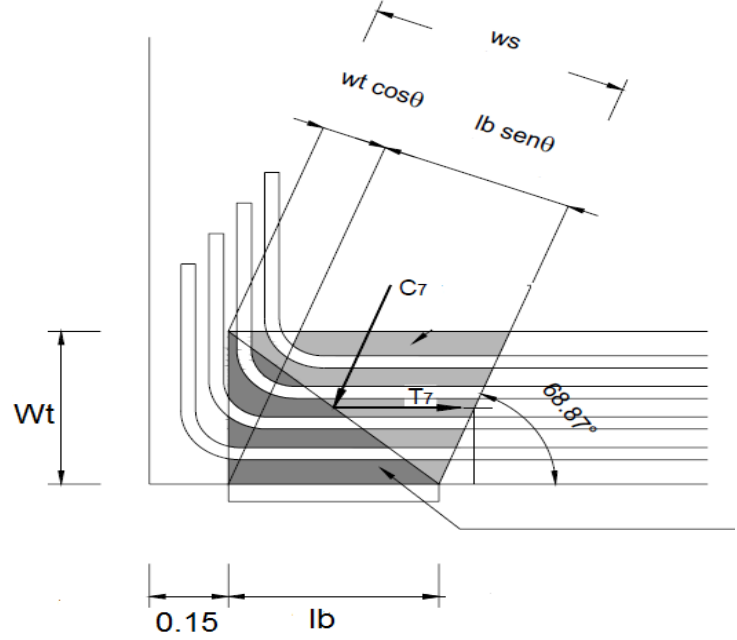

Figura 4 Zona nodal B.

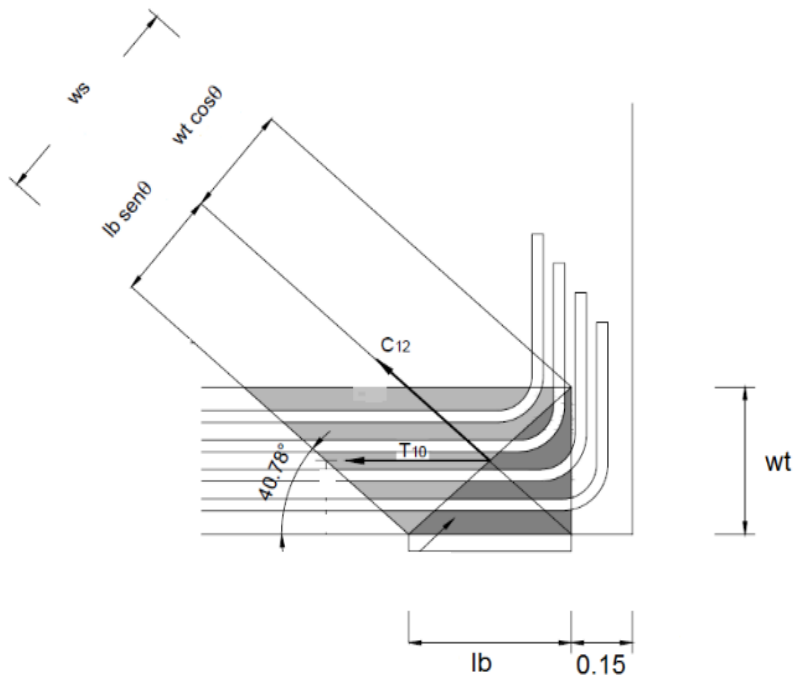

Figura 5 Zona nodal C.

Entonces con esto se concluye que el ancho de la fuerza C12, es $0.526 \mathrm{~m}$, el ancho de T10 es $0.35 \mathrm{~m}$ y el ancho de la carga es $0.40 \mathrm{~m}$. 
En este cuadro se muestra de forma resumida la verificación de las zonas nodales mencionadas.

$F_{u}$ : Fuerza última

$F_{n n}$ : Fuerza nominal

$W_{s, t}$ : Ancho de la zona nodal

$b$ : Espesor (0.35)

$f_{c u}$ : Resistencia efectiva

$f^{\prime} c$ : Resistencia a la compresión

$\beta_{n}$ :Factor de resistencia efectiva

$\emptyset$ : Factor de reducción (0.75)

\begin{tabular}{|c|c|c|c|c|c|c|c|c|c|}
\hline \multirow{2}{*}{$\begin{array}{l}\text { Nodo } \\
\text { \#Tipo }\end{array}$} & \multirow{2}{*}{ fcu } & \multicolumn{2}{|c|}{ Accion } & \multirow{2}{*}{$\begin{array}{l}\mathrm{Fu} \\
\mathrm{TN} \\
\end{array}$} & \multirow{2}{*}{$\frac{\text { Ancho }}{\mathrm{m}}$} & \multirow{2}{*}{$\begin{array}{c}\text { Fnn } \\
\text { TN }\end{array}$} & \multirow{2}{*}{$\begin{array}{c}\phi F n n \\
\mathrm{TN} \\
\end{array}$} & \multirow{2}{*}{ ¿VERIFICA? } & \multirow{2}{*}{$\mathrm{Fu} /(\phi . \mathrm{Fnn})$} \\
\hline & & Tipo & $\#$ & & & & & & \\
\hline \multirow{3}{*}{ A1 CCC } & \multirow{3}{*}{25.5} & Carga & F1 & 90 & 0.300 & 267.750 & 200.813 & SI & 0.4482 \\
\hline & & C & Po & 124.225 & 0.250 & 223.125 & 167.344 & SI & 0.7423 \\
\hline & & C & P1 & 153.401 & 0.378 & 337.365 & 253.024 & SI & 0.6063 \\
\hline \multirow{3}{*}{ A2 CCC } & \multirow{3}{*}{25.5} & Carga & $\mathrm{F} 2$ & 90 & 0.300 & 267.750 & 200.813 & $\mathrm{SI}$ & 0.4482 \\
\hline & & C & Po & 124.225 & 0.250 & 223.125 & 167.344 & SI & 0.7423 \\
\hline & & C & P2 & 153.401 & 0.378 & 337.365 & 253.024 & SI & 0.6063 \\
\hline \multirow{3}{*}{ B CCT } & \multirow{3}{*}{20.4} & C & R1 & 120 & 0.400 & 285.600 & 214.200 & SI & 0.5602 \\
\hline & & C & P7-8 & 128.65 & 0.499 & 356.286 & 267.215 & SI & 0.4814 \\
\hline & & $\mathrm{T}$ & T7 & 46.377 & 0.350 & 249.900 & 187.425 & SI & 0.2474 \\
\hline \multirow{3}{*}{ С ССТ } & \multirow{3}{*}{20.4} & C & $\mathrm{R} 2$ & 60 & 0.400 & 285.600 & 214.200 & SI & 0.2801 \\
\hline & & C & P12 & 91.866 & 0.526 & 375.564 & 281.673 & SI & 0.3261 \\
\hline & & $\mathrm{T}$ & $\mathrm{T} 10$ & 69.565 & 0.350 & 249.900 & 187.425 & SI & 0.3712 \\
\hline \multirow{4}{*}{ D CTT } & \multirow{4}{*}{15.3} & $\mathrm{C}$ & P11 & 91.866 & 0.363 & 194.387 & 145.790 & $\mathrm{SI}$ & 0.6301 \\
\hline & & $\mathrm{T}$ & T6 & 60 & 0.150 & 80.325 & 60.244 & SI & 0.9960 \\
\hline & & $\mathrm{T}$ & T9 & 139.13 & 0.350 & 187.425 & 140.569 & SI & 0.9898 \\
\hline & & $\mathrm{T}$ & $\mathrm{T} 10$ & 69.565 & 0.350 & 187.425 & 140.569 & $\mathrm{SI}$ & 0.4949 \\
\hline
\end{tabular}

Figura 6 Verificación de Nodos.

\section{Verificación de los puntales}

Los puntales inclinados se consideran con forma de botella, según el código $\mathrm{ACl}$, entonces por ello cabe resaltar de que los puntales horizontales y verticales se consideran de forma prismática, y los inclinados de botella. Las áreas necesarias se verán en el siguiente cuadro resumen. $\left(F_{u}\right.$ :Fuerza última del tirante)

\begin{tabular}{lrr}
\hline Tirante & Fu / Tn & Ast necesaria/ $\mathrm{cm} 2$ \\
\hline T1 & 66.953 & 21.255 \\
T2 & 57.273 & 18.182 \\
T3 & 10.896 & 3.459 \\
T4 & 30 & 9.524 \\
T5 & 30 & 9.524 \\
T6 & 60 & 19.048 \\
T7 & 46.377 & 14.723 \\
T8 & 92.754 & 29.446 \\
T9 & 139.13 & 44.168 \\
T10 & 69.565 & 22.084 \\
\hline
\end{tabular}

Figura 7 Fuerza en los tirantes y área necesaria en cada tirante.

Tirante 1:

1era capa: 2 barras de $11 / 8: 2 * 6.41=12.82 \mathrm{~cm} 2$

2 da capa : 2 barras de $11 / 8: 2 * 6.41=\underline{12.82 \mathrm{~cm} 2}$

$25.64 \mathrm{~cm} 2$

Tirante 2 y 3 :

1era capa: 2 barras de $1: 2 * 5.1=10.2 \mathrm{~cm} 2$

2 da capa : 2 barras de $1: 2 * 5.1=\underline{10.2 \mathrm{~cm} 2}$

$20.4 \mathrm{~cm} 2$

Tirante 4 y 5 :

Se podría pensar en distribuir esta armadura en una zona de longitud igual a: $L=z^{*} \operatorname{cotg} \theta$.

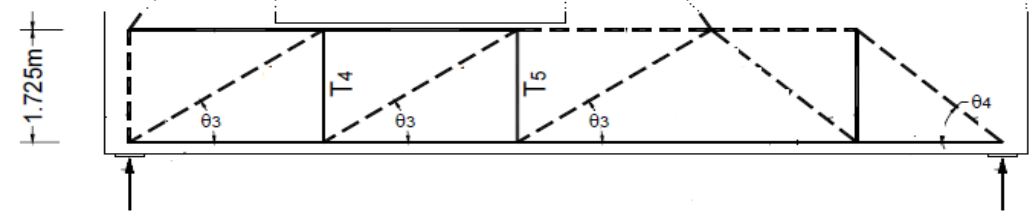

Figura 8 Tirante 4 y 5 . 
En la muestra se tiene: $z=1.725 \mathrm{~m}$ y $\theta=32.898^{\circ}$, por lo que resulta una longitud igual a $2.667 \mathrm{~m}$. La armadura necesaria será entonces igual a: $9.52 \mathrm{~cm} 2 / 2.667 \mathrm{~m}=3.57 \mathrm{~cm} 2 / \mathrm{m}$

\section{Utilizar armadura vertical $\varphi 1 / 2 @ 25 \mathrm{~cm}$ en ambas caras.}

Tirante 6:

Se podría pensar en distribuir esta armadura en una zona de longitud igual a: $L=z^{*} \operatorname{cotg} \theta$.

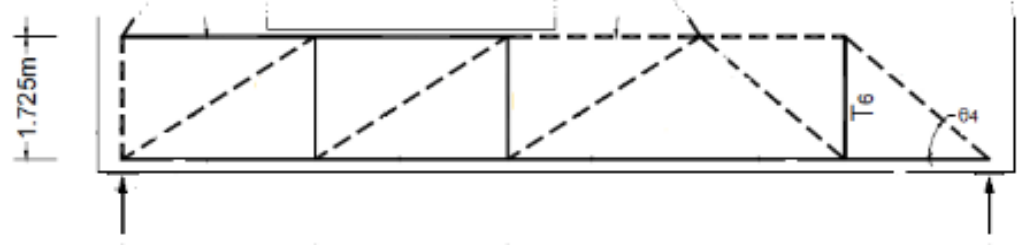

Figura 9 Tirante 6.

En la muestra se tiene: $z=1.725 \mathrm{~m}$ y $\theta=40.778$ o por lo que resulta una longitud igual a $2.000 \mathrm{~m}$. La armadura necesaria será entonces igual a: $19.05 \mathrm{~cm} 2 / 2.000 \mathrm{~m}=9.525 \mathrm{~cm} 2 / \mathrm{m}$

\section{Utilizar armadura vertical $\varphi 1 / 2 @ 25 \mathrm{~cm}$ en ambas caras.}

Tirante $7,8,9$ y 10 :

1era capa: 2 barras de 1: $2 * 5.1=10.2 \mathrm{~cm} 2$

2 da capa : 2 barras de $1: 2 * 5.1=10.2 \mathrm{~cm} 2$

3era capa: 2 barras de 1: $2 * 5.1=10.2 \mathrm{~cm} 2$

4ta capa : 2 barras de $1: 2 * 5.1=\underline{10.2 \mathrm{~cm} 2}$

$40.8 \mathrm{~cm} 2$

Área de refuerzo vertical y horizontal

Área de refuerzo vertical $=25 \mathrm{~cm}$

Esto corresponde a barras de $\mathbf{1 / 2}$ " cada $\mathbf{2 5} \mathrm{cm}$, cumple ya que la cuantía para el refuerzo vertical es 0.003 , según el código $\mathrm{ACl}$.

Área de refuerzo horizontal $=44 \mathrm{~cm}$

En ambos casos espaciamiento máximo no debe exceder entre $\mathrm{d} / 5$ y $30 \mathrm{~cm}$. Esto corresponde a barras de $1 / 2$ " cada $30 \mathrm{~cm}$, cumple ya que la cuantía para el refuerzo horizontal es 0.003 , según el código $\mathrm{ACl}$.

g. Resultado final de Armadura de la viga de gran peralte según la técnica puntal-tirante.

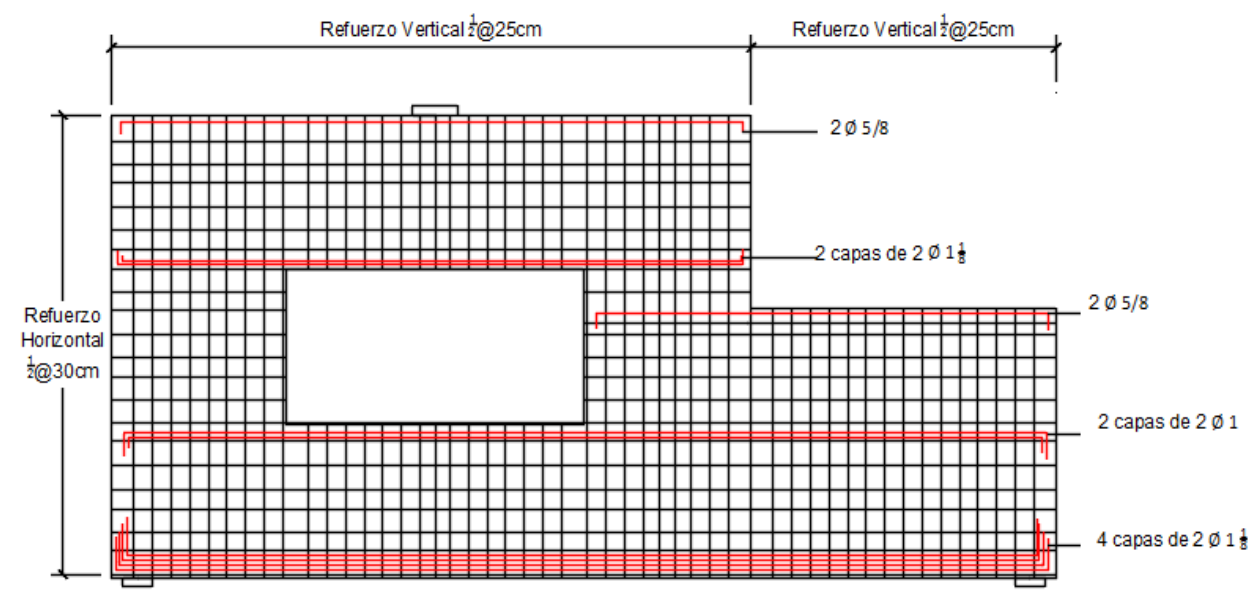

Figura 10 Armado de acero de refuerzo de acero de la muestra. 
A traves de la siguiente imagen se hizo tres cortes en la estructura para poder visualizar a con mas detalle la distribucion de acero en la misma.

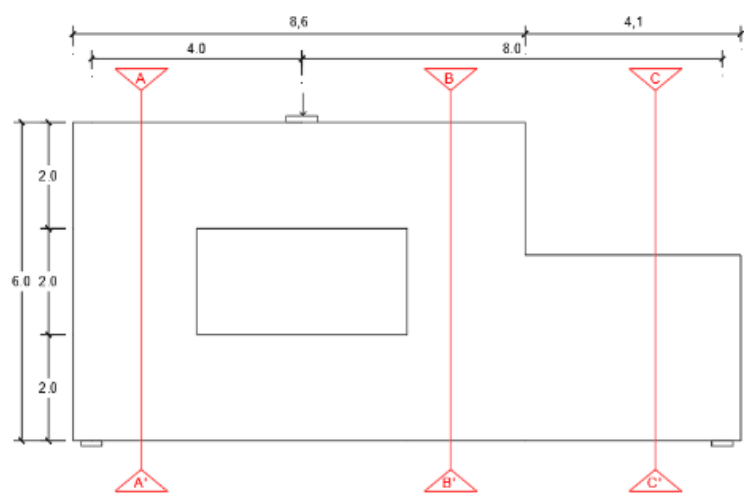

Figura 11 Corte A, B y C de la viga de gran peralte.

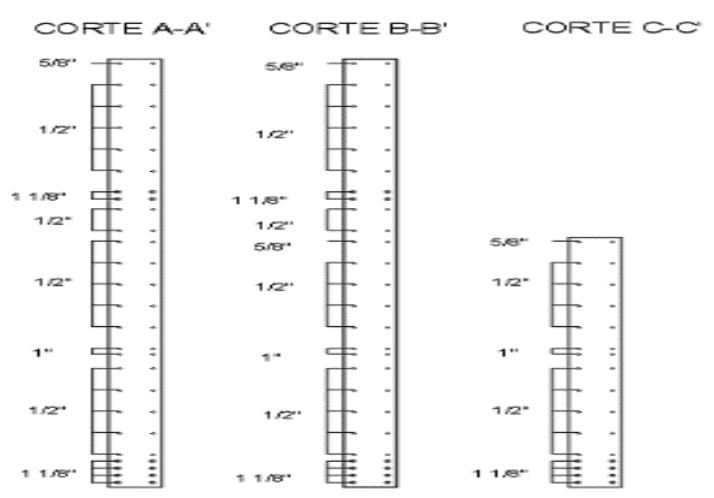

Figura 12 Refuerzo de acero según los cortes.

\section{Verificación de la técnica con la herramienta de diseño AStrutTIE}

Con esta herramienta se corrobora las fuerzas obtenidas manualmente, con los datos de la geometría de estructura, puntales y tirantes, datos de geometría de nodos, dimensiones de puntales y tirantes, propiedades de los nodos, fuerzas en elementos y reacciones, anchos efectivos de puntales y tirantes, esfuerzo y relación de esfuerzos de puntales tirantes y zonas nodales

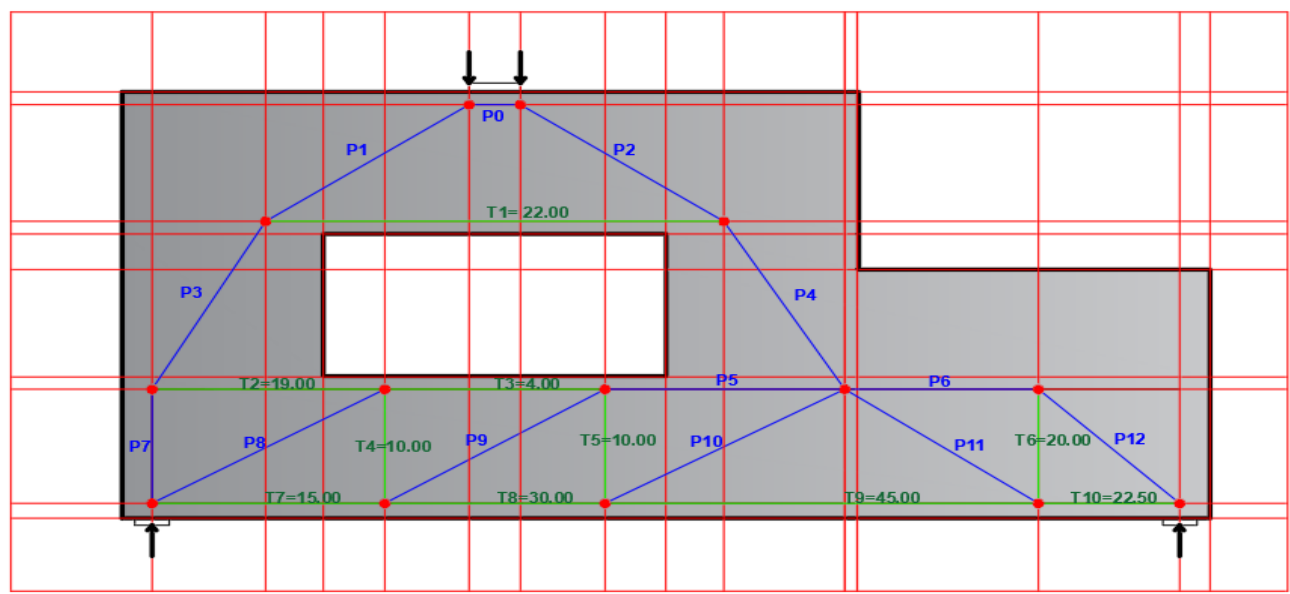

Figura 13 Arreglo de la armadura y sección de acero provisto.

\section{Diseño según la norma e.060}

- Cálculo de fuerzas en la muestra. $R_{1}=120, R_{2}=60$

Verificación por corte.

Según la Norma Técnica Peruana, menciona que la cortante nominal tiene que ser mayor que la cortante última. $V_{n}=911.812 \mathrm{Tn}$

Siendo 120Tn el valor de nuestra cortante ultima, cumple la verificación por corte.

- Área de acero horizontal y vertical.

Área de acero horizontal: $s=87.23 \mathrm{~cm}$

Según la Norma Peruana no puede ser menor de d/5 y mayor $d 30 \mathrm{~cm}$, los cálculos tuvieron en cuenta estos parámetros para la elección del espaciado, cumpliendo con la Norma Técnica Peruana. $S 1=30.00 \mathrm{~cm}$ 


\section{El área de acero horizontal será varillas de $1 / 2$ espaciado cada $30 \mathrm{~cm}$.}

Área de acero vertical: $s=52.34 \mathrm{~cm}$

Según la Norma Peruana no puede ser menor de d/5 y mayor $d 30 \mathrm{~cm}$, los cálculos tuvieron en cuenta estos parámetros para la elección del espaciado, cumpliendo con la Norma Técnica Peruana. $S 1=30.00 \mathrm{~cm}$

\section{INSTRUMENTOS}

El instrumento que se usó en la investigación es el software de elementos finitos Abaqus Cae, para ello se pondrá las consideraciones que se tuvo en el modelado.

\section{Parte}

El concreto se modela como un sólido deformable 3D, para el comportamiento del concreto se utiliza un modelo constitutivo, a elección del diseñador, para esta investigación se usó el modelo de Hognestad, para el comportamiento a compresión y el Euro Código para el comportamiento a tracción del concreto. En las consideraciones de borde o apoyos, se optó por poner placas que simulen el comportamiento de los apoyos reales. (García G. L., 2019)

\section{Propiedades}

En las propiedades se inserta el comportamiento elástico y plástico del concreto y del acero refuerzo, además de sus características, como el módulo de elasticidad y el módulo de poisson.

Tabla 5

\begin{tabular}{lc} 
Valores del Software Abaqus Cae. \\
\hline \multicolumn{2}{c}{ PLASTICITY } \\
\hline Dilation angle & 30 \\
\hline Eccentricity & 0.1 \\
\hline Fb0/fc0 & 1.16 \\
\hline k & 0.6667 \\
\hline Viscosity Parameter & 0.0001 \\
\hline
\end{tabular}

\section{Mesh}

Una malla o también conocida como mesh, es una red de líneas que se conectan entre sí con una cierta cantidad de nodos utilizados para resolver numéricamente el problema en juego bajo cargas externas. Es la base de cada producto numérico de elementos finitos es la discretización del modelo considerado en una serie de elementos. (García G. L., 2019).

En esta investigación se usó tres casos de mesh, uno de 25, 20 y de 15, donde se optó por usar el de 15 ya que sus resultados son más precisos. (Lu, 2014).

\section{Carga}

La viga de concreto reforzado se estudian bajo dos tipos de carga, la capacidad de carga límite de servicio, que se usa para el control de la desviación y la carga de falla final, que se usa para el diseño, además de esto se dispuso 2 cargas amplificadas para ver como falla la estructura.

Tabla 6

Carga en la viga de gran peralte con alto nivel de asimetría.

\begin{tabular}{ccc}
\hline Carga de diseño & Carga 1 & Carga 2 \\
\hline $180 \mathrm{Tn}$ & $360 \mathrm{Tn}$ & $720 \mathrm{Tn}$ \\
\hline
\end{tabular}

\section{Análisis}

Se va a considerar la geometría no lineal. Para grandes desplazamientos, necesitamos actualizar nuestra matriz de rigidez después de cada incremento de carga; por lo tanto, se utiliza la tensión y las tensiones verdaderas en lugar de las tensiones y tensiones nominales. 


\section{RESULTADOS}

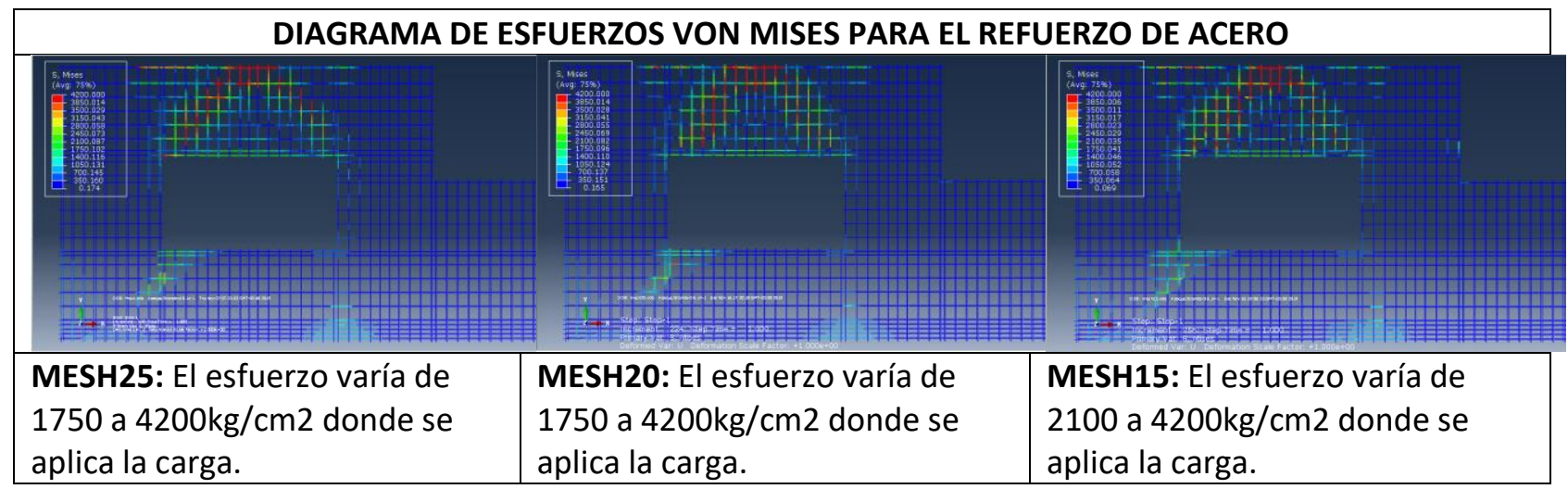

Figura 14 Diagrama de esfuerzos Von Mises para el refuerzo de acero.

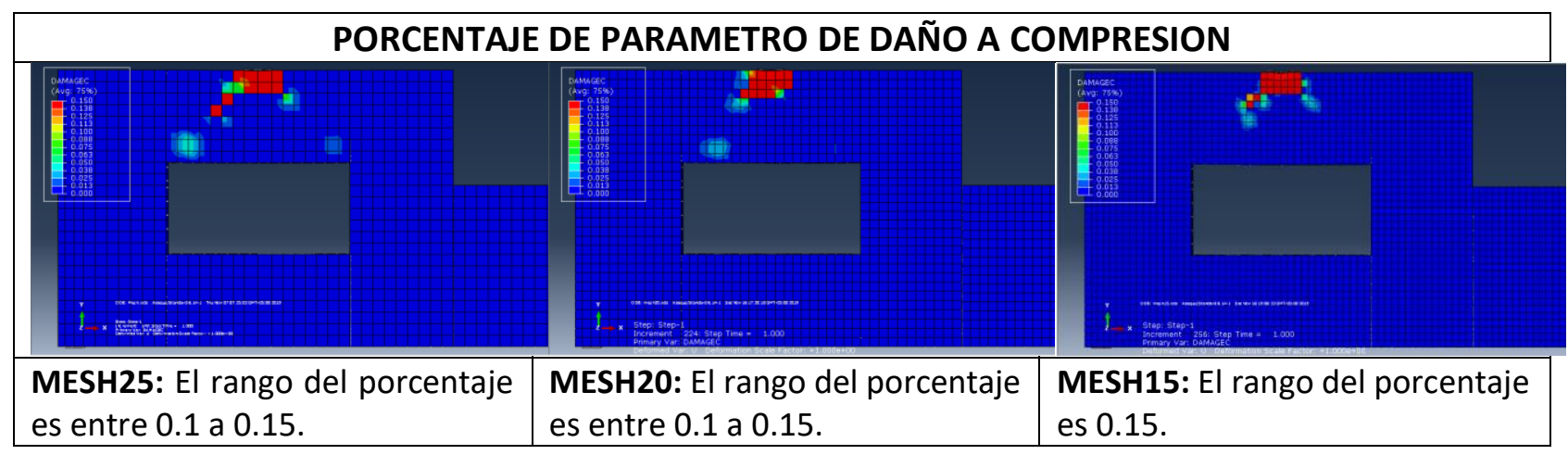

Figura 15 Porcentaje de parámetro de daño a compresión.

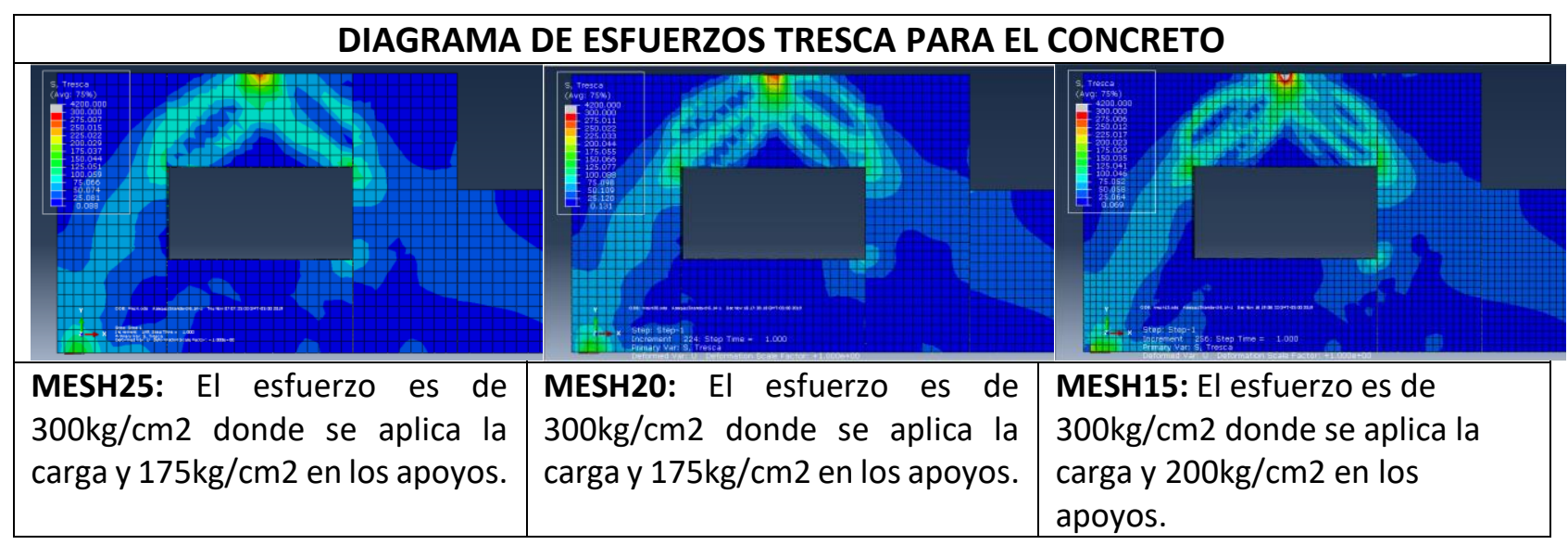

Figura 16 Diagrama de esfuerzos Tresca para el concreto.

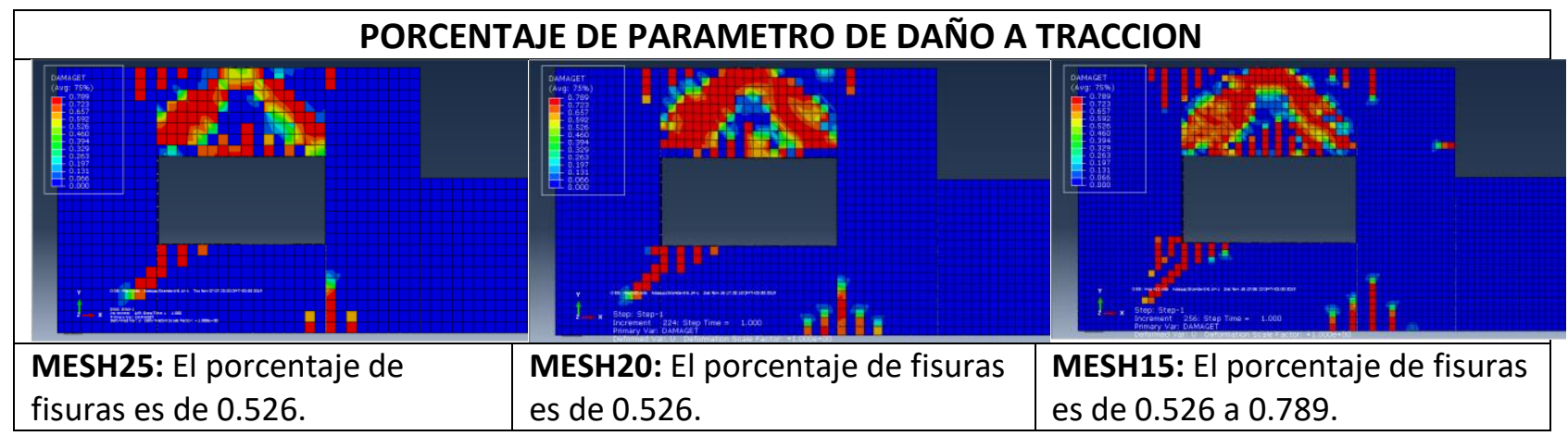

Figura 17 Porcentaje de parámetro de daño a tracción. 


\section{DISCUSIÓN}

Con estos resultados se puede comprobar que la técnica puntal-tirante es eficiente para la determinación del diseño de elementos estructurales con alto nivel de asimetría. Con los resultados de los diagramas de Von Mises del acero de refuerzo se pudo verificar que la técnica puntal-tirante o strut and tie se comporta de manera adecuada con respecto a la distribución acero de refuerzo a través de la técnica ya mencionada. Se pudo demostrar de que las regiones $B$ (Teorema de Navier) y las regiones $D$ que no cumplen este teorema, modelan adecuadamente la concentración de esfuerzos, a través de esta técnica ya que esta, identifica a cada una de estas para el diseño estructural. La técnica puntal tirante es la técnica más adecuada para diseño de vigas de gran peralte con alto nivel de asimetría de concreto, ya que su diseño se basa en las regiones discontinuas tanto geométricas y estáticas. El software de elementos finitos Abaqus Cae demostró que con el diseño de la técnica puntal tirante la viga de gran peralte asimétrica tiene un buen comportamiento estructural, resistiendo la carga idealizada. Se recomienda estudiar criterios para la idealización de propuestas de armadura de la estructura de concreto armado que vayan a diseñar. Al ser la técnica puntal tirante un método cinemático, por lo mismo de que se obtienen modelos diferentes para cada caso, se recomienda estudiar la superposición de estos casos para obtener la cantidad de refuerzo de acero para que resistan la carga solicitada, eso aplicado en estructuras simétricas y asimétricas.

\section{CONCLUSIONES}

- La técnica puntal-tirante es un método para el diseño de estructuras discontinuas de concreto armado, alternativo a los métodos empíricos (Norma Peruana de Concreto Armado E.060) o mediante elementos finitos que se utilizan en su resolución. El resultado de la utilización de esta técnica es la determinación de la cantidad de refuerzo de acero de la estructura de concreto armado, de tal manera que resista las cargas a la cual es sometido.

- La determinación de las Regiones D (Discontinuas), aplica a cualquier discontinuidad, tanto en geometría o tipo de carga que posea la estructura; en contraposición a métodos netamente empíricos, que son válidos para magnitudes específicas. Identificar estas regiones es vital para cualquier tipo de diseño.

- La aplicación de técnica puntal tirante a una viga de gran peralte con alto nivel de asimetría demanda una gran cantidad de acero de refuerzo, exactamente son 12 varillas de acero de 1 $1 / 8^{\prime \prime}, 4$ varillas de $1^{\prime \prime}, 4$ varillas de 5/8", además de refuerzo horizontal de $1 / 2 @ 30 \mathrm{~cm}$ y un refuerzo vertical de $1 / 2 @ 25 \mathrm{~cm}$, esto se debe a que esta técnica considera las discontinuidades de la estructura, y la muestra estudiada es $100 \%$ discontinua, el aplastamiento ocurre en la zona donde se aplica la carga, y las fisuras se presentan en la misma dirección de los puntales.

- La verificación a través del software Abaqus Cae precisó el esfuerzo de Von Mises de 2100 a $4200 \mathrm{~kg} / \mathrm{cm} 2$, un esfuerzo de Tresca de $300 \mathrm{~kg} / \mathrm{cm} 2$ en la zona de la carga aplicada y $200 \mathrm{~kg} / \mathrm{cm} 2$ en los apoyos, un porcentaje de parámetro de daño a compresión o aplastamiento de 0.15 , un porcentaje de parámetro de daño a tracción o fisuras en un rango de 0.526 a 0.789 , y un desplazamiento de -2.198 en la zona de la carga y de -0.727 en la parte media de la viga.

\section{REFERENCIAS BIBLIOGRÁFICAS}

Adm, H. (2017). Modelado de estructuras de concreto por Puntal Tirante.

Aedo, M. A. (2004). Modelo Puntal-Tensor Aplicado al Diseño de Elementos de Hormigon Armado.

America Concrete Institute. (Enero de 2002). Reglamento Estructural para edificaciones. Codigo ACI 318.

America. 
America Concrete Institute. (Enero de 2002). Reglamento Estructural para Edificaciones. Codigo ACl 318. America.

American Concrete Institute. (Enero de 2002). Reglamento Estructural para Edificaciones. Codigo ACl 318. America.

Arkiplus. (2019). Arkiplus. Obtenido de https://www.arkiplus.com/concreto-armado/: https://www.arkiplus.com/concreto-armado/

Bernoulli, N. (1782).

Camacho, J. A. (2010). Diseño de vigas altas con modelos a escala en micro. Bogota.

Civiles, R. I. (2015). Vigas de gran peralte.

Contreras, J. C. (2010). PROYECTO DE ESTRUCTURAS DE UN EDIFICIO EN SURCO.

Expok. (s.f.). expoknews. Obtenido de https://www.expoknews.com/las-ciudades-verticales-son-larespuesta-sustentable-al-crecimiento-urbano/

García, G. A. (2009 de ABRIL de 2009). Modelamiento puntal tensor para muros cortos. Modelamiento puntal tensor para muros cortos. Chile.

García, G. L. (17 de Julio de 2019). Comportamiento de vigas de gran peralte empleando tecnicas modernas exigiendo la norma peruana de concreto armado. Comportamiento de vigas de gran peralte empleando tecnicas modernas exigiendo la norma peruana de concreto armado. Tacna, Perú.

Loayza, D. R. (2015). Resistencia probable a flexocompresion de muros estructurales de concreto armado. Resistencia probable a flexocompresion de muros estructurales de concreto armado.

Lu, W. Y. (2014). Vigas de gran peralte de concreto armado.

Metals. (2019). Simulacion numérica de concreto reforzado.

RNE. (2009). Reglamento Nacional de Edificaciones. Ley 27792.

S.R.Chowdhury. (26 de Diciembre de 2014). Numerical Modelling of Reinforced Concrete Beams with Opening. Numerical Modelling of Reinforced Concrete Beams with Opening. C, Chittagong.

Sotomayor Medina, A. M. (2019). DESARROLLO DEL MÉTODO DE PUNTAL TIRANTE. Lima.

Torres, M. (2018). Método de Puntal Tirante.

Wikipedia. (20 de Mayo de 2017). Obtenido de https://es.wikipedia.org/wiki/Tensi\%C3\%B3n_de_Von_Mises

Wikipedia. (9 de Diciembre de 2018). Obtenido de https://es.wikipedia.org/wiki/Deformaci\%C3\%B3n Young, N. S. (Febrero de 2009). Deep Beam Design Using Strut-Tie Model. Korea. 